\title{
Brief Intermittent Cocaine Self-Administration and Abstinence Sensitizes Cocaine Effects on the Dopamine Transporter and Increases Drug Seeking
}

\author{
Erin S Calipari ${ }^{1,2}$, Cody A Siciliano ${ }^{2}$, Benjamin A Zimmer ${ }^{2,3}$ and Sara R Jones*,2 \\ 'Fishberg Department of Neuroscience and Friedman Brain Institute, Icahn School of Medicine at Mount Sinai, New York, NY, USA; ${ }^{2}$ Department \\ of Physiology and Pharmacology, Wake Forest School of Medicine, Winston-Salem, NC, USA; ${ }^{3}$ Department of Neuroscience, The Medical \\ University of South Carolina, Charleston, SC, USA
}

\begin{abstract}
Although traditional sensitization paradigms, which result in an augmentation of cocaine-induced locomotor behavior and dopamine (DA) overflow following repeated experimenter-delivered cocaine injections, are often used as a model to study drug addiction, similar effects have been difficult to demonstrate following cocaine self-administration. We have recently shown that intermittent access (IntA) to cocaine can result in increased cocaine potency at the DA transporter (DAT); however, traditional sensitization paradigms often show enhanced effects following withdrawal/abstinence periods. Therefore, we determined a time course of IntA-induced sensitization by examining the effects of I or 3 days of IntA, as well as a 7-day abstinence period on DA function, cocaine potency, and reinforcement. Here we show that cocaine potency is increased following as little as 3 days of IntA and further augmented following an abstinence period. In addition, IntA plus abstinence produced greater evoked DA release in the presence of cocaine as compared with all other groups, demonstrating that following abstinence, both cocaine's ability to increase DA release and inhibit uptake at the DAT, two separate mechanisms for increasing DA levels, are enhanced. Finally, we found that IntA-induced sensitization of the DA system resulted in an increased reinforcing efficacy of cocaine, an effect that was augmented after the 7-day abstinence period. These results suggest that sensitization of the DA system may have an important role in the early stages of drug abuse and may drive the increased drug seeking and taking that characterize the transition to uncontrolled drug use. Human data suggest that intermittency, sensitization, and periods of abstinence have an integral role in the process of addiction, highlighting the importance of utilizing pre-clinical models that integrate these phenomena, and suggesting that IntA paradigms may serve as novel models of human addiction.

Neuropsychopharmacology (20I5) 40, 728-735; doi:I 0.I038/npp.20I4.238; published online 8 October 20I4
\end{abstract}

\section{INTRODUCTION}

Repeated drug use in humans has been shown to result in sensitization to cues predicting drug availability and drug effects, which are thought to drive the continued drug use that leads to addiction; thus, understanding the neurochemical process that mediates these behaviors may give important insights into the mechanisms that drive the process of addiction (Vezina and Leyton, 2009). Rodent models of sensitization have been suggested to be a translational model of addiction (Robinson and Becker, 1986; Post, 1980), but these paradigms employ non-contingent forms of administration, and neurochemical sensitization has been difficult to demonstrate with contingent paradigms, such as intravenous self-administration, that better

*Correspondence: Dr SR Jones, Department of Physiology and Pharmacology, Wake Forest School of Medicine, Medical Center Boulevard, Winston-Salem, NC 27I57, USA, Tel: 336 716 8533, Fax: 336716 8501, E-mail: srjones@wakehealth.edu

Received 16 June 2014; revised 20 August 2014; accepted 21 August 2014; accepted article preview online 12 September 2014 model human abuse patterns. For example, extended-access cocaine self-administration has been shown repeatedly to reduce cocaine potency at the dopamine transporter (DAT) and produce concomitant behavioral tolerance (Calipari et al, 2013a, b, c, d; Calipari et al, 2014a, b; Ferris et al, 2011, 2012, 2013a,b; Oleson et al, 2011; Hurd et al, 1989). The behavioral and neurochemical consequences of cocaine exposure are dependent upon a number of factors including, frequency of administration, intermittency, and abstinence (Jones et al, 1996: Vezina and Leyton, 2009; Bouayad-Gervais et al, 2014; Calipari et al, 2013a), prompting recent work with intermittent access (IntA) self-administration to explore sensitized cocaine effects (Calipari et al, 2013d; Zimmer et al, 2012).

IntA is a novel self-administration paradigm that restricts cocaine intake to brief ( $5 \mathrm{~min}$ ) access periods every $30 \mathrm{~min}$ (Zimmer et al, 2012). IntA was recently shown to produce sensitization of cocaine's effects at the DAT, which is the first time that the sensitization of cocaine's effects on the DAT have been observed using contingent administration (Calipari et al, 2013d). Although this work showed that sensitization of cocaine effects at the DAT occurred $24 \mathrm{~h}$ 
following 14-day IntA, the temporal profile of these effects remains to be determined. Further, in traditional experimenterdelivered cocaine paradigms, intermittency and deprivation are important aspects of the sensitization process, and often, sensitized cocaine responses are not expressed until after a period of abstinence (Pierce and Kalivas, 1997; Robinson and Becker, 1986; Post, 1980). Thus it is possible that the increases in cocaine potency that occur immediately following IntA may incubate during abstinence.

The aim of this study was to determine the temporal profile of the sensitization of cocaine effects at the DAT during the early stages of IntA self-administration, assess the effects of a period of abstinence on cocaine potency and the reinforcing efficacy of cocaine. Here we show that IntA results in sensitization of both cocaine potency and dopamine (DA) signaling (Calipari et al, 2013a, d). These effects were observable after as little as 3 days of exposure to IntA cocaine self-administration, and a 7-day abstinence period resulted in further sensitization of cocaine's effects. The neurochemical changes were accompanied by behavioral changes, where IntA-induced increases in the reinforcing efficacy of cocaine were further enhanced following an abstinence period. Here we show that IntA-induced sensitization is characteristic of many of the behavioral and neurochemical consequences of addiction in humans, suggesting that IntA models may serve as an ideal model for studying the changes that occur during the transition to cocaine addiction.

\section{MATERIALS AND METHODS}

\section{Animals}

Male Sprague-Dawley rats (375-400 g; Harlan Laboratories, Frederick, Maryland), maintained on a $12: 12 \mathrm{~h}$ reverse light/dark cycle (0300 hours lights off; 1500 hours lights on) with food and water ad libitum.

\section{Self-Administration}

Rats were anesthetized and implanted with chronic indwelling jugular catheters as previously described (Calipari et al, 2013d). Animals were singly housed, and all sessions took place in the home cage during the active/dark cycle (09001500 hours). Animals underwent a training paradigm within which animals were given access on a fixed ratio one (FR1) schedule to a cocaine-paired lever, which, upon responding, initiated an intravenous injection of cocaine $(0.75 \mathrm{mg} / \mathrm{kg}$, infused over $4 \mathrm{~s}$ ). After each response/infusion, the lever was retracted and a stimulus light was illuminated for a 20-s timeout period. Training sessions were terminated after a maximum of 20 infusions or $6 \mathrm{~h}$, whichever occurred first. Acquisition criteria were characterized by 20 injections administered for two consecutive days with consistent interinfusion intervals.

\section{IntA}

During each 6-h session, animals had access to cocaine for 12 five-minute trails separated by 25 -minute timeout periods. Within each 5 -min session, there were no timeouts other than during each infusion, and the animal could press the lever on an FR1 schedule to receive a 1-s infusion of cocaine $(0.375 \mathrm{mg} / \mathrm{kg} / \mathrm{inf})$. Animals received either 1 or 3 consecutive days of IntA self-administration before voltammetry or threshold experiments. A separate group of animals underwent 3 days of IntA self-administration followed by a 7-day abstinence period during which they had no access to the self-administration lever.

\section{Controls}

All animals were compared with control animals that underwent catheter surgery and lived in the same housing conditions as self-administering animals.

\section{In Vitro Voltammetry}

Animals were killed for fast-scan cyclic voltammetry experiments the morning following the final self-administration session $(\sim 18 \mathrm{~h})$, or on the seventh day of abstinence. Tissue was prepared as described previously (Calipari et al, 2013b; Siciliano et al, 2014). A carbon fiber (Goodfellow Corporation, Coraopolis, PA) microelectrode (100-200 $\mu \mathrm{M}$ length, $7 \mu \mathrm{M}$ radius) and bipolar stimulating electrode were placed into the core of the NAc. DA release was evoked by a single electrical pulse $(350 \mu \mathrm{A}, 4 \mathrm{~ms}$, monophasic) applied to the tissue every $5 \mathrm{~min}$. Extracellular DA was recorded by applying a triangular waveform $(-0.4$ to +1.2 to $-0.4 \mathrm{~V} v s \mathrm{Ag} / \mathrm{AgCl}$, $400 \mathrm{~V} / \mathrm{s})$. Once the extracellular DA response was stable, cocaine $(0.03-30 \mu \mathrm{mol} / \mathrm{l})$ was applied cumulatively to the brain slice. To evaluate DA kinetics and drug potency, evoked levels of DA were modeled using Michaelis-Menten kinetics. For cocaine concentration-response curves, app. $K_{\mathrm{m}}$, a measure of apparent affinity for the DAT, was used to determine changes in the ability of cocaine to inhibit DA uptake.

\section{Threshold}

In a separate group of animals, the threshold procedure was used to determine IntA-induced changes in cocaine selfadministration. The threshold procedure was performed before IntA and then again following 3 days of IntA or following 3 days of IntA and a 7-day abstinence period. The threshold procedure is a behavioral economics approach to assessing drug taking/seeking and reinforcing efficacy. The threshold procedure consists of giving rats access to a descending series of 11 unit doses of cocaine (421, 237, 133, $75,41,24,13,7.5,4.1,2.4$, and $1.3 \mu \mathrm{g}$ /injection) available on an FR1 schedule of reinforcement. Each dose is available for $10 \mathrm{~min}$, with each bin presented consecutively across the 110 -min session. Animals performed this procedure for 3 consecutive days and responding was averaged to derive the values used. Completion of the procedure produces a within-session dose-response curve, depicted in Figure 4a. During the initial bins of the procedure, when the dose is high, the animal is able to obtain a preferred level of cocaine intake with minimal responding. As the dose is lowered across bins, the animal must increase responding to maintain consistent intake, until the dose becomes low enough that preferred levels of cocaine cannot be maintained and responding decreases. Shifts in responding across the dose-response curve can be analyzed using behavioral economics principles, as described below. 
Behavioral economic analysis was used to determine the parameters of maximal price paid $\left(P_{\max }\right)$ and consumption at a minimally constraining price $\left(Q_{0}\right)$, as described previously (Oleson et al, 2011; Oleson and Roberts, 2009; Oleson and Roberts, 2012). Briefly, $P_{\max }$ and $Q_{0}$ values were derived mathematically using a demand curve. Demand curves were generated by curve-fitting individual animals' intake using an equation: $\log (Q)=\log \left(Q_{0}\right)+k \times(e-\alpha \times$ $Q_{0} \times C-1$ ) (Hursh and Silberberg, 2008; Christensen et al, 2008). In this equation, $P_{\max }$ was determined to be the unit price at which the first derivative point slope of the function $=-1$ (Hursh and Winger, 1995). The value $k$ was set to 2 for all animals, whereas $Q_{0}$ and $\alpha$, which represent the acceleration of the function in response to changing price, were estimated to achieve best fit (Hursh and Silberberg, 2008; Christensen et al, 2008). These measures are explained in detail below.

$Q_{0}: Q_{0}$ is a measure of the animals' preferred level of cocaine consumption. This can be measured when the dose is high and cocaine is available at low effort, or a minimally constraining price. This preferred level of consumption is established in the early bins of the threshold procedure.

$P_{\text {max }}$ : Price is expressed as the responses emitted to obtain $1 \mathrm{mg}$ of cocaine, thus as the dose is decreased in each consecutive bin of the threshold procedure, price increases. As the session progresses, animals must increase responding on the active lever in order to maintain stable intake. $P_{\max }$ is the price at which the animal no longer emits enough responses to maintain intake and consumptions decreases. Thus, animals with higher $P_{\max }$ will increase responding to maintain cocaine levels farther into the doseresponse curve; in other words they will pay a higher price for cocaine. Previous work has demonstrated that $P_{\max }$ is highly correlated with break points on a progressive ratio schedule of reinforcement, confirming that the threshold procedure accurately assesses reinforcing efficacy (Oleson et al, 2011).

\section{Calculating $\mathrm{K}_{\mathbf{i}}$ Values}

Inhibition constants $\left(\mathrm{K}_{\mathrm{i}}\right)$ were determined by plotting the linear concentration-effect profiles and determining the slope of the linear regression. The $\mathrm{K}_{\mathrm{i}}$ was calculated by the equation $\mathrm{K}_{\mathrm{m}}$ /slope.

\section{Statistics}

Graph Pad Prism (version 5, La Jolla, CA, USA) was used to statistically analyze data sets and create graphs. Data are presented as mean \pm SEM and percentage unless otherwise stated. Baseline voltammetry data and $K_{i}$ values were compared using a one-way analysis of variance (ANOVA). When main effects were obtained $(P<0.05)$, differences between groups were tested using a Tukey post hoc test. Release data and data obtained after perfusion of cocaine were subjected to a two-way ANOVA with experimental group and concentration of drug as the factors. Differences between groups were tested using a Bonferroni post hoc test. Correlational analyses were used to assess the association of DA release in the presence of drug with cocaine potency, as measured by both app. $\mathrm{K}_{\mathrm{m}}$ and $\mathrm{K}_{\mathrm{i}}$. Pearson's correlation coefficients were used to measure the strength of correla-

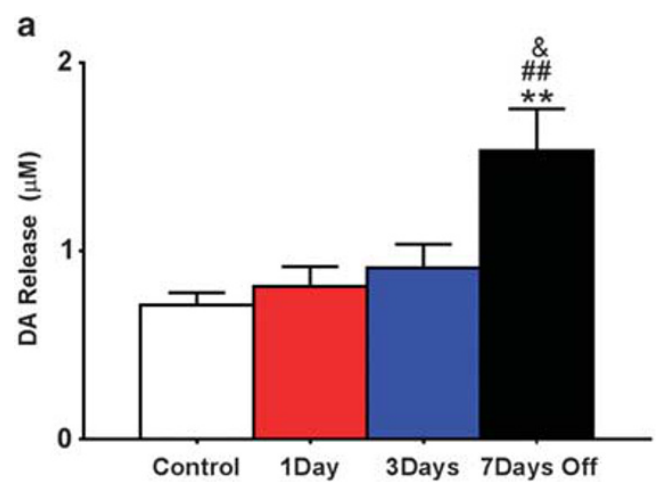

b

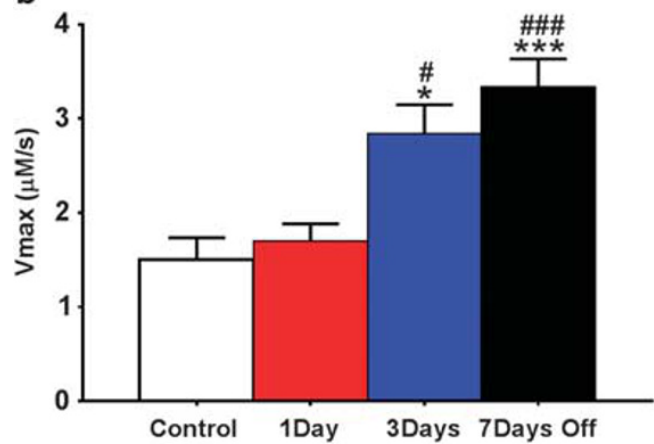

Figure I Intermittent access (IntA) cocaine self-administration alters presynaptic dopamine system kinetics. (a) Stimulated dopamine (DA) release in $\mu \mathrm{M}$ between groups. Animals underwent IntA cocaine selfadministration for I or 3 days. One group was given 3 days of IntA with 7 days off. (b) Maximal rate of DA uptake $\left(V_{\max }\right)$ across groups. Data are expressed as mean $( \pm$ SEM). $* p<0.05$ vs control; $* * p<0.01$ vs control; **** $p<0.00$ I vs control; ${ }^{\&} p<0.05$ vs IntA 3 day; ${ }^{\#} p<0.05$ vs IntA I day;

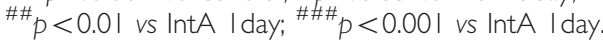

tion. $P_{\max }$ and $Q_{0}$ values were analyzed pre and post IntA were compared using a paired Student's $t$-test. All $p$-values of $<0.05$ were considered to be statistically significant.

\section{RESULTS}

Increased Stimulated DA Release and $V_{\max }$ following 3-Day IntA are Augmented by an Abstinence Period

A one-way ANOVA indicated a main effect of the selfadministration group on stimulated DA release $\left(F_{3,27}=\right.$ $6.17, p<0.01$; Figure 1a). Although release was not significantly elevated following 1 or 3 days of IntA cocaine self-administration, Tukey post hoc analysis revealed that stimulated release was elevated following a 3-day IntA with a 7-day abstinence period as compared with controls $(q=5.24, p<0.01), 1$ day of IntA $(q=4.99, p<0.01)$ and 3 days of IntA $(q=4.16, p<0.05)$.

A one-way ANOVA indicated a main effect of the selfadministration group on $V_{\max }\left(\mathrm{F}_{3,27}=11.24, p<0.0001\right.$; Figure $1 \mathrm{~b})$. Tukey post hoc analysis revealed that maximal rate of uptake was elevated in the 3-day IntA group as compared with controls $(q=4.85, p<0.05)$ and 1 day of IntA $(q=4.31, p<0.05)$. Further, a 7 -day abstinence period following the 3 -day self-administration period resulted in increased uptake relative to controls $(q=6.83, p<0.001)$ and 1 day of IntA $(q=6.37, p<0.001)$, however, uptake was not 


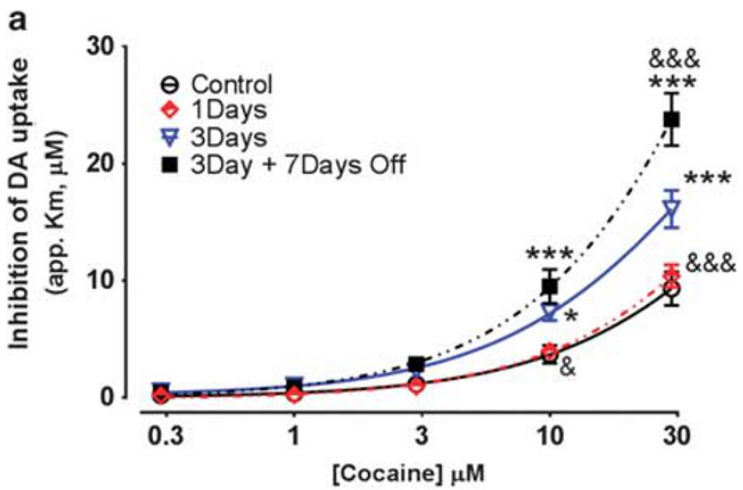

b

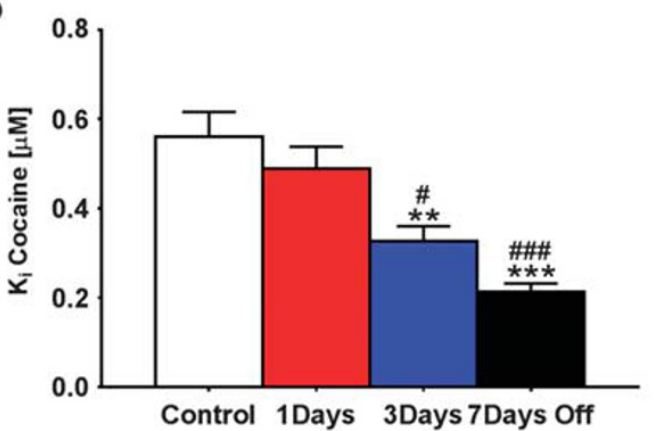

Figure 2 Intermittent access (IntA) self-administration results in sensitization to the neurochemical effects of cocaine. (a) Cumulative cocaine $(0.3-30 \mu \mathrm{M})$ dose-response curves in slices containing the nucleus accumbens core. Cocaine potency is increased following 3 days of IntA and augmented further following a 7-day abstinence period. (b) Bar graph of $K_{i}$ values for cocaine in control, IntA, and IntA with abstinence groups. $K_{i}$ values are a measure of the concentration of drug at which $50 \%$ inhibition is achieved. Data are expressed as mean $( \pm$ SEM). $* 0<0.05$ vs control; *** $p<0.0$ I vs control; $* * * * * 0.001$ vs control; ${ }^{*} p<0.05$ vs IntA 3 day;

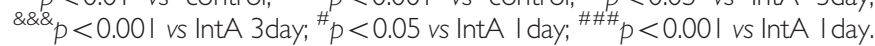

significantly different from the 3-day self-administration group that had no abstinence period.

\section{Increased Cocaine Potency following 3-Day IntA is} further Augmented by a 7-Day Abstinence Period

A two-way repeated measures ANOVA revealed a main effect of self-administration history on cocaine potency $\left(\mathrm{F}_{4}\right.$, $100=12.68, p<0.001$; Figure 2a). Although there was no significant difference between 1-day IntA and control animals, Bonferroni post hoc analysis revealed that 3-day IntA cocaine self-administration resulted in increased cocaine potency as compared with control animals at the $10 \mu \mathrm{M}(t=2.93, p<0.05)$ and $30 \mu \mathrm{M}(t=5.54, p<0.001)$ concentrations. In addition, 3-day IntA cocaine selfadministration resulted in increased cocaine potency as compared with 1 -day IntA animals at the $10 \mu \mathrm{M}(t=3.53$, $p<0.01)$ and $30 \mu \mathrm{M}(t=6.11, p<0.001)$ concentrations. Animals that underwent 3-day IntA cocaine self-administration, and a 7-day abstinence period had increased cocaine potency as compared with control animals at the $10 \mu \mathrm{M}(t=4.60, p<0.001)$ and $30 \mu \mathrm{M}(t=11.44, p<0.001)$ concentrations; 1 -day IntA animals at the $10 \mu \mathrm{M}(t=4.39$, $p<0.001)$ and $30 \mu \mathrm{M}(t=10.64, p<0.001)$ concentrations; and 3-day IntA animals at the $30 \mu \mathrm{M}(t=5.35, p<0.001)$ concentration.
$K_{i}$ is a measure of the drug concentration that reduces uptake to $50 \%$ of its uninhibited value; thus a decrease in $\mathrm{K}_{\mathrm{i}}$ is indicative of increased potency. A one-way ANOVA revealed a significant main effect of the group on $\mathrm{K}_{\mathrm{i}}$ $\left(\mathrm{F}_{3,28}=13.96, p<0.0001\right.$; Figure $\left.2 \mathrm{~b}\right)$. Tukey post hoc analysis revealed that $K_{i}$ was reduced in the 3-day IntA group relative to controls $(q=5.58, p<0.01)$ and 1 -day IntA $(q=4.18, p<0.05)$. A 7 -day abstinence period resulted in a further decrease in $\mathrm{K}_{\mathrm{i}}$ relative to controls $(q=8.09$, $p<0.001)$ and 1 -day IntA $(q=6.88, p<0.0001)$.

\section{Cocaine-Induced Increases in DA Release are Enhanced following Int $\mathrm{A}$ and Abstinence}

In addition to determining the effects of cocaine directly at the DAT following IntA and abstinence, we also assessed the effects of cocaine self-administration on cocaine-induced increases in stimulated DA release in the NAc core. A twoway ANOVA revealed a main effect of cocaine concentration on stimulated DA release $\left(\mathrm{F}_{5},{ }_{26}=38.31, p<0.001\right.$; Figure 3a). In addition, there was a main effect of selfadministration history on stimulated DA release $\left(F_{3,26}=\right.$ 7.19, $p<0.001)$. Bonferroni post hoc analysis revealed significant increases in cocaine-induced increases in DA release following IntA plus abstinence at the $0.3(p<0.001)$, $1(p<0.001), 3(p<0.001)$, and $10 \mu \mathrm{M}(p<0.001)$ concentrations as compared with control animals. In addition, there were increases in cocaine-induced DA release following IntA plus abstinence as compared with IntA 1-day selfadministration at the $0.3(p<0.05), 1(p<0.01), 3(p<0.05)$, and $10 \mu \mathrm{M}(p<0.05)$ concentrations. Finally, there were increases in cocaine-induced DA release following IntA plus abstinence as compared with 3-day IntA at the 0.3 $(p<0.05), 1(p<0.01)$, and $3 \mu \mathrm{M}(p<0.01)$ concentrations. Further, there was a significant concentration-self-administration interaction $\left(\mathrm{F}_{15,26}=7.19, p<0.05\right)$.

\section{Cocaine Potency is not Correlated with Measures of Release}

In order to confirm that differences in stimulated release were not driving the IntA-induced changes in cocaine potency at the DAT, we correlated stimulated DA release in the presence of cocaine with $\mathrm{K}_{\mathrm{i}}$ (Figure $3 \mathrm{~b}$ ). We found that stimulated release in the presence of cocaine did not correlate with uptake inhibition (Control, $r=-0.48$, NS; IntA 1 day, $r=-0.55$, NS; IntA 3 days, $r=0.07$, NS; IntA 7 days off, $r=0.06, \mathrm{NS})$, indicating that the magnitude of evoked release is not a significant factor that influences uptake inhibition and that the two are in fact separate phenomena.

\section{Increased Reinforcing Efficacy of Cocaine following IntA was Augmented after a 7-Day Abstinence Period}

To determine the effects of IntA-induced sensitization of the DA system and cocaine potency on the reinforcing efficacy of cocaine, a separate group of animals performed the threshold procedure at two time points, once following acquisition of cocaine responding (Figure 4a, left panel, representative animal) and again following either 3 days of IntA alone or 3 days of IntA plus a 7-day abstinence period (Figure $4 \mathrm{a}$, right panel, representative animal). In the 3 -day 

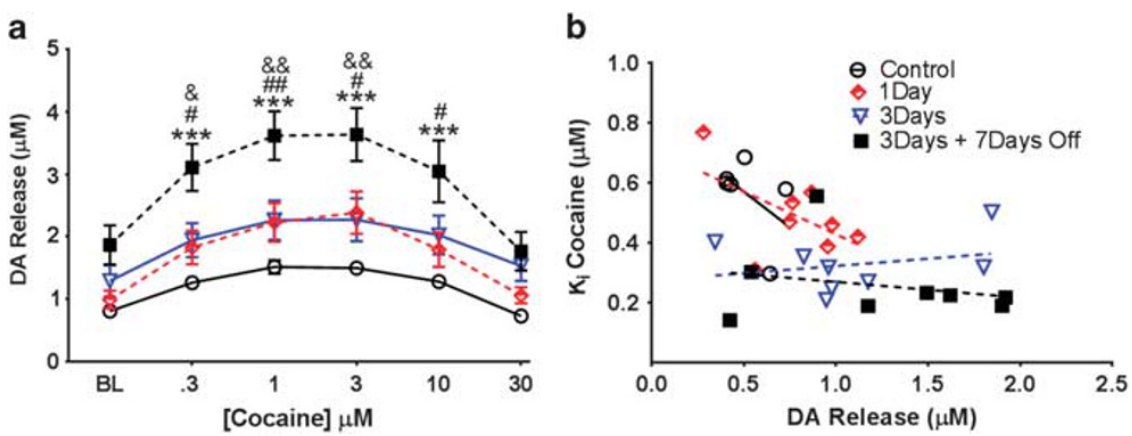

Figure 3 Drug-induced dopamine (DA) release is enhanced following intermittent access (IntA) and abstinence. (a) Stimulated DA release, measured across all cocaine concentrations for control, I-day IntA, 3-day IntA, and 3-day IntA with a 7-day abstinence period. IntA plus abstinence enhanced DA release in the presence of cocaine as compared with both control groups and other self-administration groups. (b) Correlational analysis showing no relationship between DA release in the presence of cocaine $(30 \mu \mathrm{M})$ and cocaine potency as measured by the $K_{i}$ of cocaine for the transporter. Data are

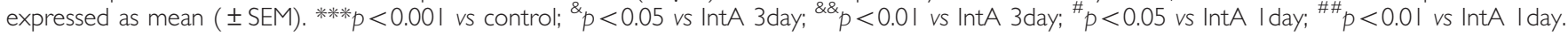

IntA group, a Student's $t$-test revealed a significant increase in $P_{\max }$ post IntA as compared with baseline $\left(t_{9}=2.21\right.$, $p<0.05$; Figure $4 \mathrm{~b}$ ), indicating that IntA increases the reinforcing efficacy of cocaine. Similarly, a Student's $t$-test revealed a significant increase in $P_{\max }$ following 3 days of IntA plus a 7-day abstinence period $\left(\mathrm{t}_{6}=3.11, p<0.05\right.$; Figure $4 \mathrm{c}$ ). In addition, a Student's $t$-test revealed that the increase in $P_{\max }$ was significantly greater in the 7-day abstinence group, indicating that IntA-induced increases in the reinforcing efficacy of cocaine augment over an abstinence period $\left(t_{15}=2.25, p<0.05\right.$; Figure $\left.4 \mathrm{~d}\right)$.

\section{Cocaine Consumption is Reduced following IntA and Abstinence}

In addition to determining the reinforcing efficacy of cocaine, the threshold procedure also measures $Q_{0}$, a measure of consumption. In the 3-day IntA group, there was no change in $Q_{0}$ (Figure $4 \mathrm{e}$ ), indicating that although IntA increases $P_{\max }$, it does not change the preferred dose that the animal will consume. Conversely, a Student's $t$-test revealed a significant decrease in $Q_{0}\left(t_{6}=3.80, p<0.01\right.$; Figure $4 \mathrm{f}$ ) following 3 days of IntA plus a 7-day abstinence period. A between-group comparison revealed that the animals that were given a 7-day abstinence period showed a greater reduction in $Q_{0}$ than IntA animals with no abstinence $\left(t_{13}=1.78, \quad p<0.05\right.$; Figure $\left.4 \mathrm{~g}\right)$. It has been shown previously that when drug is available at a low response requirement, animals titrate their intake around a preferred brain cocaine level, thought to be determined by the subjective effects of the compound. Because the subjective effects of cocaine are dependent on the DA system, sensitization of cocaine's effects on DA neurotransmission causes animals to titrate around a lower level.

Together, these data indicate that IntA alters the reinforcing properties of cocaine in regard to both motivation as well as consumption, and that the magnitude of these effects is enhanced by a withdrawal period.

\section{DISCUSSION}

This study demonstrates that intermittent cocaine selfadministration results in distinct neurochemical alterations that affect not only DA system function but also the potency and reinforcing efficacy of cocaine. There has been a great deal of work aimed at aligning rodent self-administration with human addiction pathology, including escalation, punished responding, and extinction/reinstatement/relapse paradigms (Ahmed and Koob, 1999, 1998; Jonkman et al, 2012; Shaham et al, 2003). Here we highlight the importance of the temporal profile of cocaine self-administration and the consideration of abstinence periods when choosing a translationally relevant model, as compensatory mechanisms associated with drug exposure are highly dependent on both the pattern of self-administration and withdrawal. Because rodent models cannot encompass all aspects of human drug addiction, it is particularly important to select models that accurately model discrete aspects of the processes. Intermittent patterns of cocaine self-administration are characteristic of human intake patterns and the IntA model provides a novel paradigm for contingent studies on the sensitization that occurs within the human addiction process.

In our previous work, and many currently accepted cocaine self-administration models, emphasis has been placed on maximizing an animal's intake, with the thought that more intake results in greater neurochemical effects and more accurately models the profile of human cocaine abuse. Here we demonstrate that that is not the case, as high, continuous intake is not necessary to produce robust neurochemical effects. The sensitization of cocaine potency at the DAT, observed in the current study, is opposite from the well-documented decrease in cocaine potency after extended-access cocaine self-administration (Hurd et al, 1989; Ferris et al, 2011, 2012, 2013a, b; Calipari et al, 2013c, $\mathrm{d}$; Calipari et al, 2014a) and suggests that cocaine is more effective at elevating DA in the NAc core following brief intermittent usage, and that an abstinence period increases the effects. The NAc core is involved in reinstatement of drug seeking after periods of abstinence (Millan et al, 2011), and increased cocaine potency in this region may promote increased rewarding and reinforcing effects of cocaine, potentially leading to greater risk of compulsive or addictive-like cocaine intake. Indeed, we show that the motivation to self-administer cocaine is significantly increased following IntA. Thus, it is possible that intermittent use of cocaine in humans results in a sensitized 
a
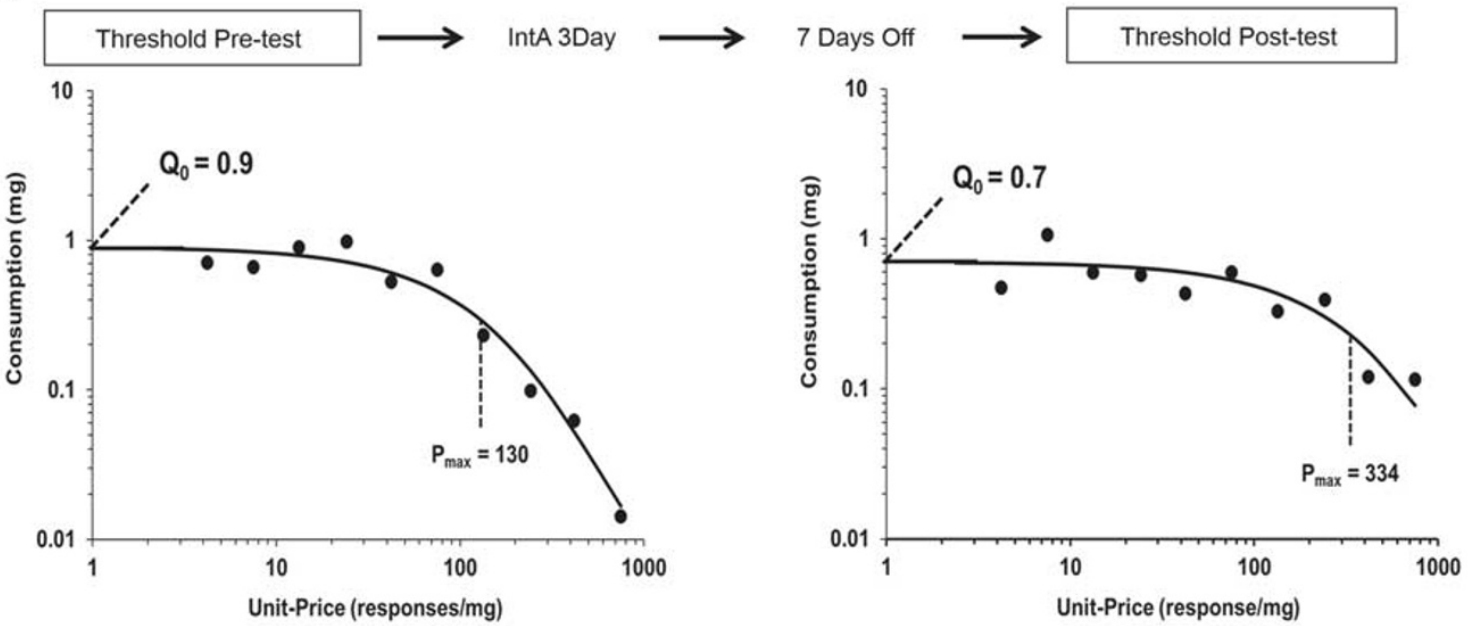

b

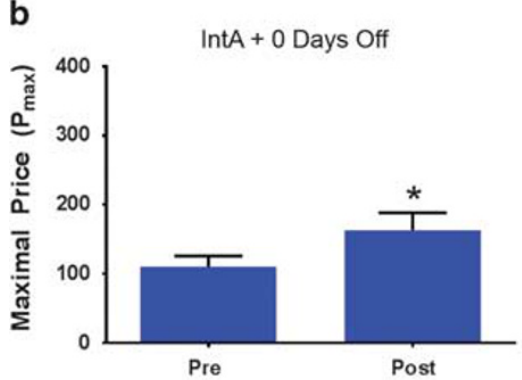

e

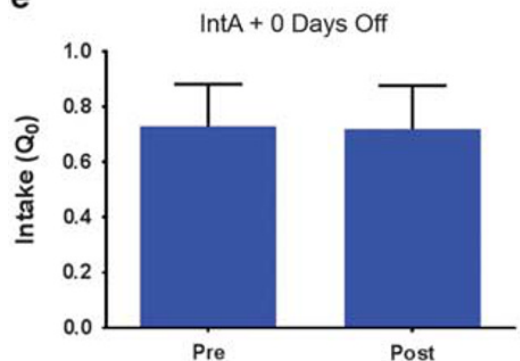

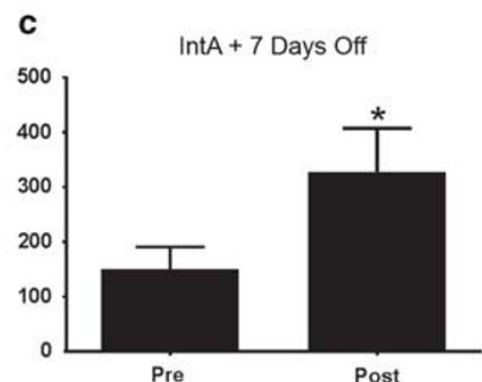

f

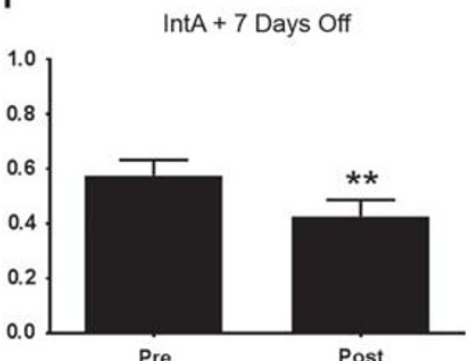

d

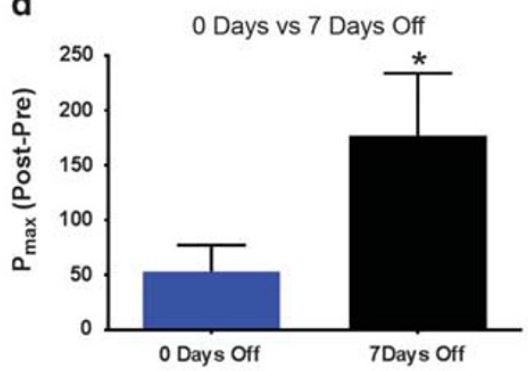

g

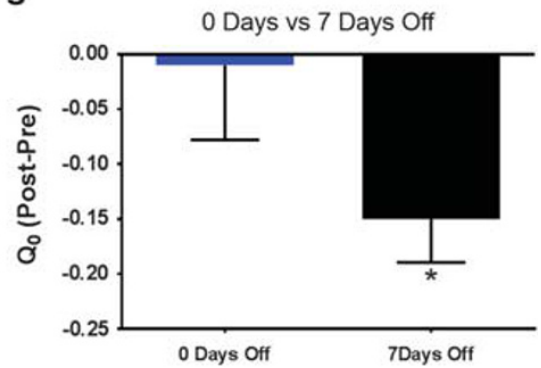

Figure 4 Cocaine reinforcement is augmented by intermittent access (IntA) and further enhanced by abstinence. Effects of 3-day IntA and 3-day IntA plus 7 days of abstinence on $P_{\max }(b-d)$ and cocaine consumption at a minimally constraining price $\left(Q_{0} ; \mathrm{e}-\mathrm{g}\right)$ as compared with a pre-IntA. (a) Representative demand curves showing pre-IntA threshold assessment (left) and following 3 days of IntA access plus a 7-day abstinence period (right). (b) Three-day IntA produced a significant increase in the maximal price $\left(P_{\max }\right)$ paid for cocaine. (c) Three-day IntA followed by a 7-day withdrawal period produced a significant increase in the maximal price $\left(P_{\max }\right)$ paid for cocaine. (d) A 7-day withdrawal period produced a significant increase in the maximal price $\left(P_{\text {max }}\right)$ paid for cocaine as compared with 3-day IntA alone. (e) Three-day IntA did not change cocaine consumption when minimally constrained by price ( $\left.Q_{0}\right)$. ( $f$ ) Threeday IntA followed by a 7-day withdrawal period reduced $Q_{0}$. (g) A 7-day withdrawal period produced a significant reduction in the $Q_{0}$ for cocaine as compared with 3-day IntA alone. Data are expressed as mean ( \pm SEM) $P_{\max }$ or consumption. ). $* 0<0.05$ vs pre-IntA; *** $p<0.0$ I vs pre-IntA.

cocaine response that promotes the transition into sustained use and addiction.

It has been suggested that humans administer cocaine in an intermittent pattern, rather than maintaining stable levels, highlighting the importance of determining the neurochemical and behavioral consequences of cocaine's effects when administered in a similar pattern (Beveridge et al, 2012). Traditional pre-clinical models of cocaine addiction using self-administration paradigms rely on prolonged access to cocaine over many days. Escalation during long-access (LgA) self-administration has long been postulated to model the switch from recreational drug use to addiction (Koob and Le Moal, 1997). However, the sustained cocaine intake associated with LgA may not accurately model human intake patterns and produces opposite neurochemical consequences, as compared with IntA (Calipari et al, 2013a). Here we show that IntA for only 3 days was sufficient to increase both uptake rates and cocaine's ability to inhibit the DAT. IntA also produced concomitant increases in the reinforcing efficacy of cocaine, suggesting that intermittent use of cocaine drives subsequent drug use, potentiating the process leading to addiction. This finding, paired with the increased motivation to administer cocaine after 7 days of withdrawal, suggests that animals become more sensitive to the effects of cocaine overall following IntA and withdrawal. The 
enhanced cocaine potency in the NAc likely drives the increased reinforcing efficacy of cocaine following IntA. Indeed, here we show that at time points when cocaine potency is increased, $P_{\max }$, a measure of reinforcing efficacy, is also increased. Further, because the effort an animal is willing to expend to obtain drug $\left(P_{\max }\right)$ increases during withdrawal, these adaptations may have a role in relapse following extended periods of abstinence.

In regard to relapse, sustained high-level use of cocaine over long periods of time is not often observed in cocaine addicts, but rather, cocaine users cycle between repeated periods of cocaine abuse followed by abstinence and relapse (Cohen and Sas, 1994). This usage pattern highlights the importance of understanding the neurochemical changes that occur during the withdrawal period. IntA followed by a 7 -day abstinence period resulted in further sensitization of (1) cocaine-induced augmentation of evoked DA release, (2) cocaine potency at the DAT, and (3) the reinforcing efficacy of cocaine, as compared with IntA alone. The effects of cocaine on evoked DA release were not related to the changes in uptake inhibition, as demonstrated by a lack of correlation between the two measures. Cocaine-induced increases in DA release have been shown previously to occur via a synapsin-dependent mechanism, and are independent of cocaine's ability to inhibit the DAT (Venton et al, 2006). Here we show that IntA augments both cocaine's effects on the DAT as well as its effects on exocytotic DA release, and cocaine-induced release and enhanced uptake inhibition may act synergistically to increase DA levels and drive the increased motivation to administer cocaine at this time point. The effects of a withdrawal period on cocaine's neurochemical and behavioral effects are consistent with many in vivo studies, demonstrating that experimenter-delivered cocaine sensitization paradigms with withdrawal/abstinence periods result in increased stimulant and cocaine-induced DA overflow as measured by microdialysis (Kalivas and Duffy, 1993; Parsons and Justice, 1993; Robinson and Becker, 1982; Robinson et al, 1988). Further, we observed an enhancement of cocaine reinforcement following IntA and withdrawal, and it is possible that the changes in cocaine potency and cocaine-induced increases in evoked DA release may converge to drive the enhanced reinforcing effects of cocaine and relapse following withdrawal.

In humans, a drug abuse history is postulated to be associated with greater activation of the ventral striatum by drug-associated cues, and the duration of drug abuse is positively correlated with the magnitude of activation (Prisciandaro et al, 2014). Here we show that following a 3-day history of IntA cocaine self-administration, evoked DA release was unchanged. However, a 7-day abstinence period after IntA resulted in augmented stimulated DA release, in the absence of cocaine, as compared with controls. The increased DA release could point to increases in the releasable DA pool, which may enhance release during phasic signaling events. Increased release during phasic DA neuron firing in response to stimuli in the environment could lead to enhanced cue-reward associations, and facilitate relapse following periods of abstinence (Ostlund et al, 2014).

This work highlights the importance of choosing clinically relevant and translational models for specific aspects of human drug abuse. Although LgA may serve as a better model for the tolerance that develops after chronic heavy cocaine intake in humans, IntA better models the sensitization to motivational aspects of drug abuse, as well as sensitized responses to cues predicting drug availability. Taken together, these data show that intermittency and withdrawal have essential roles in determining the neurochemical and behavioral consequences of cocaine selfadministration. It is suggested that in humans, drug use occurs under conditions of limited availability, which may lead individuals to administer drug in an intermittent pattern (Ahmed et al, 2013), and the available human literature supports these assertions. Further, the neurochemical and behavioral effects of cocaine in humans are sensitized for long periods, up to years, following drug abuse, and are thought to drive the intermittent cycles of relapse and drug administration that are characteristic of stimulant addiction (Prisciandaro et al, 2014). Thus, because the temporal profile of cocaine administration and abstinence periods have profound effects on the neurochemical and behavioral consequences of cocaine administration, models of cocaine abuse should attempt to accurately take into account the patterns of cocaine administration by human cocaine addicts. Together, the human data demonstrate that intermittency, sensitization, and periods of abstinence and relapse converge to further the addiction process, highlighting the importance of considering these aspects in the determination of the most translationally relevant pre-clinical models.

\section{FUNDING AND DISCLOSURE}

This work was funded by NIH grants R01 DA024095, R01 DA030161, R01 DA014030, P50 DA006634 (SRJ), T32 DA007246 and F31 DA031533 (ESC), and T32 AA007565 (CAS). The authors declare no conflict of interest.

\section{ACKNOWLEDGEMENTS}

We would like to thank Dr Amanda Gabriele for her help with the self-administration studies in the current manuscript.

\section{REFERENCES}

Ahmed SH, Koob GF (1998). Transition from moderate to excessive drug intake: change in hedonic set point. Science 282: 298-300.

Ahmed SH, Koob GF (1999). Long-lasting increase in the set point for cocaine self-administration after escalation in rats. Psychopharmacology (Berl) 146: 303-312.

Ahmed SH, Lenoir M, Guillem K (2013). Neurobiology of addiction versus drug use driven by lack of choice. Curr Opin Neurobiol 23: $581-587$.

Beveridge TJR, Wray P, Brewer A, Shapiro B, Mahoney JJ, Newton TF (2012). Analyzing Human Cocaine Use Patterns to Inform Animal Addiction Model Development. Published abstract for the College on Problems of Drug Dependence Annual Meeting. Palm Springs, CA.

Bouayad-Gervais K, Minogianis EA, Lévesque D, Samaha AN (2014). The self-administration of rapidly delivered cocaine promotes increased motivation to take the drug: contributions of 
prior levels of operant responding and cocaine intake. Psychopharmacology (Berl) (in press).

Calipari ES, Beveridge TJ, Jones SR, Porrino LJ (2013a). Persistent decreases in functional activity of limbic brain regions following extended access cocaine self-administration. Eur J Neurosci 38: 3749-3757.

Calipari ES, Ferris MJ, Caron MG, Roberts DC, Jones SR (2013b). Methylphenidate self-administration increases the potency and reinforcing effects of releasers through a dopamine transporter mechanism. Nat Commun 4: 2720.

Calipari ES, Ferris MJ, Melchior JR, Bermejo K, Salahpour A, Roberts DC et al (2014a). Methylphenidate and cocaine selfadministration produce distinct dopamine terminal alterations. Addict Biol 19: 145.

Calipari ES, Ferris MJ, Roberts DCS, Jones SR (2013c). Extended access cocaine self-administration results in tolerance to the dopamine-elevating and locomotor-stimulating effects of cocaine. J Neurochem 128: 224-232.

Calipari ES, Ferris MJ, Siciliano CA, Jones SR (2014b). Intermittent cocaine self-administration produces sensitization of stimulant effects at the dopamine transporter. J Pharmacol Exp Ther 349: 192-198.

Calipari ES, Ferris MJ, Zimmer BA, Roberts DCS, Jones SR (2013d). Temporal pattern of cocaine intake determines tolerance versus sensitization of cocaine effects at the dopamine transporter. Neuropsychopharmacology 38: 2385-2392.

Christensen C, Silberberg A, Hursh S, Huntsberry M, Riley A (2008). Essential value of cocaine and food in rats: tests of the exponential model of demand. Psychopharmacology 198: 221-229.

Cohen Peter, Sas Arjan (1994). Cocaine use in Amsterdam in nondeviant subcultures. Addict Res 2: 71-94.

Ferris MJ, Calipari ES, Mateo Y, Melchior JR, Roberts DC, Jones SR (2012). Cocaine self-administration produces pharmacodynamic tolerance: differential effects on the potency of dopamine transporter blockers, releasers, and methylphenidate. Neuropsychopharm 37: 1708-1716.

Ferris MJ, Calipari ES, Melchior JR, Roberts DC, España RA, Jones SR (2013b). Paradoxical tolerance to cocaine after initial supersensitivity in drug-use-prone animals. Eur J Neurosci 38: 2628-2636.

Ferris MJ, Calipari ES, Yorgason JT, Jones SR (2013a). Examining the complex regulation and drug-induced plasticity of dopamine release and uptake using voltammetry in brain slices. ACS Chem Neurosci 4: 693-703.

Ferris MJ, Mateo Y, Roberts DC, Jones SR (2011). Cocaineinsensitive dopamine transporters with intact substrate transport produced by self-administration. Biol Psychiatry 69: 201-207.

Hurd YL, Weiss F, Koob GF, And NE, Ungerstedt U (1989). Cocaine reinforcement and extracellular dopamine overflow in rat nucleus accumbens-an in vivo Microdialysis Study. Brain Res 498: 199-203.

Hursh SR, Silberberg A (2008). Economic demand and essential value. Psychol Rev 115: 186-198.

Hursh SR, Winger G (1995). Normalized demand for drugs and other reinforcers. J Exp Anal Behav 64: 373-384.

Jones SR, Lee TH, Wightman RM, Ellinwood EH (1996). Effects of intermittent and continuous cocaine administration on dopamine release and uptake regulation in the striatum: in vitro voltammetric assessment. Psychopharmacology (Berl) 126: 331-338.

Jonkman S, Pelloux Y, Everitt BJ (2012). Differential roles of the dorsolateral and midlateral striatum in punished cocaine seeking. J Neurosci 32: 4645-4650.
Kalivas PW, Duffy P (1993). Time course of extracellular dopamine and behavioral sensitization to cocaine. I. Dopamine axon terminals. J Neurosci 13: 266-275.

Koob GF, Le Moal M (1997). Drug abuse: hedonic homeostatic dysregulation. Science 278: 52-58.

Millan EZ, Marchant NJ, McNally GP (2011). Extinction of drug seeking. Behav Brain Res 217: 454-462.

Oleson EB, Richardson JM, Roberts DC (2011). A novel IV cocaine self-administration procedure in rats: differential effects of dopamine, serotonin, and GABA drug pre-treatments on cocaine consumption and maximal price paid. Psychopharmacology (Berl) 214: 567-577.

Oleson EB, Roberts DC (2009). Behavioral economic assessment of price and cocaine consumption following self-administration histories that produce escalation of either final ratios or intake. Neuropsychopharmacology 34: 796-804.

Oleson EB, Roberts DC (2012). Cocaine self-administration in rats: threshold procedures. Methods Mol Biol 829: 303-319.

Ostlund SB, Leblanc KH, Kosheleff AR, Wassum KM, Maidment NT (2014). Phasic mesolimbic dopamine signaling encodes the facilitation of incentive motivation produced by repeated cocaine exposure. Neuropsychopharmacology 39: 2441-2449.

Parsons LH, Justice JB Jr (1993). Serotonin and dopamine sensitization in the nucleus accumbens, ventral tegmental area, and dorsal raphe nucleus following repeated cocaine administration. J Neurochem 61: 1611-1619.

Pierce RC, Kalivas PW (1997). A circuitry model of the expression of behavioral sensitization to amphetamine-like psychostimulants. Brain Res Brain Res Rev 25: 192-216.

Post RM (1980). Intermittent versus continuous stimulation: effect of time interval on the development of sensitization or tolerance. Life Sci 26: 1275-1282.

Prisciandaro JJ, Joseph JE, Myrick H, McRae-Clark AL, Henderson S, Pfeifer J et al (2014). The relationship between years of cocaine use and brain activation to cocaine and response inhibition cues. Addiction (in press).

Robinson TE, Becker JB (1982). Behavioral sensitization is accompanied by an enhancement in amphetamine-stimulated dopamine release from striatal tissue in vitro. Eur J Pharmacol 85: 253-254.

Robinson TE, Becker JB (1986). Enduring changes in brain and behavior produced by chronic amphetamine administration: a review and evaluation of animal models of amphetamine psychosis. Brain Res 396: 157-198.

Robinson TE, Jurson PA, Bennett JA, Bentgen KM (1988). Persistent sensitization of dopamine neurotransmission in ventral striatum (nucleus accumbens) produced by prior experience with $(+)$-amphetamine: a microdialysis study in freely moving rats. Brain Res 462: 211-222.

Shaham Y, Shalev U, Lu L, De Wit H, Stewart J (2003). The reinstatement model of drug relapse: history, methodology and major findings. Psychopharmacology (Berl) 168: 3-20.

Siciliano CA, Calipari ES, Ferris MJ, Jones SR (2014). Biphasic mechanisms of amphetamine action at the dopamine terminal. J Neurosci 34: 5575-5582.

Venton BJ, Seipel AT, Phillips PE, Wetsel WC, Gitler D, Greengard $\mathrm{P}$ et al (2006). Cocaine increases dopamine release by mobilization of a synapsin-dependent reserve pool. J Neurosci 26: 3206-3209.

Vezina P, Leyton M (2009). Conditioned cues and the expression of stimulant sensitization in animals and humans. Neuropharmacology 56(Suppl 1): 160-168.

Zimmer BA, Oleson EB, Roberts DCS (2012). The motivation to self-administer is increased after a history of spiking brain levels of cocaine. Neuropsychopharmacology 37: 1901-1910. 